\title{
Association between dietary behavior and risk of hypertension among Japanese male workers
}

\author{
Mitsumasa Umesawa ${ }^{1,2}$, Akihiko Kitamura², Masahiko Kiyama ${ }^{2}$, Takeo Okada², Yuji Shimizu ${ }^{2,3}$, \\ Hironori Imano ${ }^{3}$, Tetsuya Ohira $^{3}$, Masakazu Nakamura ${ }^{2}$, Koutatsu Maruyama ${ }^{4}$ and Hiroyasu Iso ${ }^{3}$, \\ CIRCS Investigators
}

Dietary behavior can worsen or prevent hypertension. However, data on the association between dietary behavior and the risk of hypertension in Asians are limited. The aim of this study was to determine these associations in Japanese male workers. We conducted a prospective study of 30-71-year-old Japanese male workers in Osaka, Japan, between 2001 and 2011. The study subjects were 3486 normotensive males who were assessed for an average of 4.6 years using an annual survey. We defined hypertension by a systolic blood pressure of $\geqslant 140 \mathrm{~mm} \mathrm{Hg}$, a diastolic blood pressure of $\geqslant 90 \mathrm{~mm} \mathrm{Hg}$ and/or the use of antihypertensive medications. Dietary behavior questionnaires were included in the annual surveys. For each question on dietary behavior, we calculated the odds ratios (ORs) for the risk of hypertension using logistic regression models. We used subjects who consistently gave affirmative answers in the baseline and end-point surveys as a reference. The number of new cases of hypertension was 846 among 3486 subjects. Compared with subjects who eat meat frequently, subjects who did not eat meat frequently showed a higher risk of hypertension $(\mathrm{OR}=1.26,95 \%$ confidence interval $(\mathrm{Cl}): 1.00-1.59)$. Subjects who did not consume dairy products every day showed a higher risk of hypertension $(\mathrm{OR}=1.39,95 \% \mathrm{Cl}: 1.13-1.71)$ compared with those who did. Meat and dairy product intake was associated with the prevention of hypertension among Japanese male workers. Hypertension Research (2013) 36, 374-380; doi:10.1038/hr.2012.205; published online 10 January 2013

Keywords: dietary behavior; epidemiology; prospective study

\section{INTRODUCTION}

Hypertension is one of the most important risk factors for cardiovascular disease in the Japanese, as well as in Western populations. ${ }^{1-4}$ Therefore, it is important to determine the risk factors associated with hypertension in order to prevent hypertension and decrease the burden of cardiovascular disease. Although pharmacological treatment of hypertension is widely available, primary prevention of hypertension is desirable.

Several studies have reported that lifestyle is significantly associated with blood pressure ${ }^{5}$ and the incidence of hypertension. ${ }^{6}$ Dietary behavior seems to be an important risk factor because it is directly associated with energy intake, which in turn correlates with body weight control and nutrient intake and is associated with maintenance of the organism.

Several studies have examined dietary behavior in relation to the Mediterranean diet and the Dietary Approaches to Stop Hypertension (DASH) diet. ${ }^{5-10}$ Both prospective studies ${ }^{6-9}$ and intervention studies ${ }^{5,10}$ found an inverse association between the Mediterranean diet or the DASH diet and the risk of hypertension or blood pressure levels. The Mediterranean diet involves an abundant intake of plant foods, adequate intake of dairy products and fish, and low intake of meats. The DASH diet involves an abundant or adequate intake of plant foods, fish and low-fat dairy products, with limited intake of sugar-sweetened foods, red meat and added fats.

For the Asian populations, only limited data are available on the association of dietary behavior and the risk of hypertension or blood pressure level. A cross-sectional study of the Chinese population showed an inverse association between fruit and milk intake and the prevalence of hypertension. ${ }^{11}$ Another cross-sectional study of the Japanese population showed an inverse association between high fruit and vegetable intake and self-measured blood pressure levels. ${ }^{12}$ To our knowledge, however, there are no published prospective studies that have examined the association of dietary behavior and the risk of hypertension or blood pressure levels in an Asian population. Of course, knowledge from Western countries is beneficial to some extent for Asians. The Japanese Society of Hypertension reviewed clinical and epidemiological studies published from around the world and released the Japanese Society of Hypertension Guidelines for the Management of Hypertension in 2009. ${ }^{13}$ In these guidelines, desirable dietary behavior was suggested; however, these guidelines were mainly

${ }^{1}$ Center for Medical Sciences, Ibaraki Prefectural University of Health Sciences, Ibaraki, Japan; ${ }^{2}$ Osaka Center for Cancer and Cardiovascular Disease Prevention, Osaka, Japan; ${ }^{3}$ Department of Social and Environmental Medicine, Graduate School of Medicine, Osaka University, Suita, Japan and ${ }^{4}$ Department of Basic Medical Research and Education, Ehime University Graduate School of Medicine, Toon, Japan

Correspondence: Dr M Umesawa, Center for Medical Sciences, Ibaraki Prefectural University of Health Sciences, Ami 4669-2, Inashiki-gun, Ibaraki 300-0394, Japan. E-mail: starlight-shower@nifty.com

Received 9 May 2012; revised 4 October 2012; accepted 5 October 2012; published online 10 January 2013 
based on the DASH diet. We believe that more data are needed for Asians because an Asian diet is different from that of Western countries.

Our a priori hypothesis was that the development of hypertension is associated with dietary behavior in the Japanese population. Dietary behaviors that lead to obesity or involve high sodium intake may increase the risk of hypertension, whereas dietary behaviors that include the consumption of fruits, vegetables and dairy products may reduce the risk of hypertension. To test our hypothesis, we performed the present prospective study in Japanese male workers.

\section{METHODS}

\section{Study subjects}

The participants were 30-71-year-old (mean age: 45.0 years) male workers who were employees of six companies in the Osaka area of central Japan and underwent serial government-sponsored annual health checkups. The total number of participants was 6554 at the beginning of the study. We excluded 1743 participants because they were diagnosed with hypertension $(\geqslant 140 \mathrm{~mm} \mathrm{Hg}$ systolic blood pressure and/or $\geqslant 90 \mathrm{~mm} \mathrm{Hg}$ diastolic blood pressure and/or those who were taking antihypertensive medications) on the baseline cardiovascular disease risk survey. During the follow-up, 1142 participants (mean age: 46.8 years) dropped out of the study following a failure to complete a health checkup. Furthermore, we excluded 183 participants because their serum creatinine concentrations were not measured. Thus, data on 3486 subjects (mean age: 42.9 years) were used for this analysis. We obtained informed consent from all subjects according to the ethical guidelines for epidemiological research by the Ministry of Health, Labor and Welfare. The study was approved by the Ethics Committee of Osaka Medical Center for Health Science and Promotion.

\section{Risk factor survey}

The annual Cardiovascular Disease Risk Surveys were performed from 2001 to 2011. The arterial systolic blood pressures and fifth-phase diastolic blood pressures were measured by well-trained observers using a standard mercury sphygmomanometer on the right arm during the survey. The participants were seated quietly for at least $5 \mathrm{~min}$ before the measurement. We used the data from the first measurement because the blood pressure was not measured twice in all subjects. Individuals with hypertension included those found to have high blood pressure $(\geqslant 140 \mathrm{mmHg}$ systolic blood pressure and/or $\geqslant 90 \mathrm{~mm} \mathrm{Hg}$ diastolic blood pressure), as well as those being treated with antihypertensive medications.

With regard to potential confounders, the body mass index was calculated by dividing the weight in kilograms by the height in meters squared. The height was measured with subjects wearing socks, and the weight was measured with subjects wearing light clothing. Every participant was interviewed to determine their usual weekly alcohol consumption in go units, a traditional Japanese unit of volume equivalent to 23 grams of ethanol. We divided weekly ethanol intake by seven to calculate the average daily alcohol intake. The smoking habits and history of the subjects were also determined during the interview, as well as the history of hypertension, stroke, coronary heart disease, renal disease and the use of antihypertensive medications. The estimated glomerular filtration rate was calculated using the following formula established by the working group of the Japanese Chronic Kidney Disease Initiative: estimated glomerular filtration rate $\left(\mathrm{ml} \mathrm{min}^{-1} 1.73 \mathrm{~m}^{-2}\right)=1.94 \times$ $(\text { serum creatinine })^{-1.094} \times(\text { age })^{-0.287} .{ }^{14}$ Since 2001 , serum creatinine has been measured using the enzymatic method.

\section{Dietary behavior survey}

The dietary behavior survey was carried out as part of the annual Cardiovascular Disease Risk Survey from 2001 to 2011 using questionnaires. The questionnaires were based on a health assessment in Japanese. ${ }^{15}$ Well-trained public health nurses helped participants who had difficulty in answering the questionnaires. The survey consisted of 19 items related to dietary behavior (Table 1). Subjects answered either 'yes' or 'no.' We examined the reproducibility of the questionnaire by using data from 2251 male subjects who

\section{Table 119 questions of dietary behavior survey}

1. Do you have breakfast?

2. Do you have a meal just before bedtime?

3. Do you eat until you are full?

4. Do you eat between meals or before bedtime every day?

5. Do you consume soft drinks every day?

6. Do you have fried food every day?

7. Do you have one or more eggs every day?

8. Do you have meat frequently?

9. Do you have fish or shellfish more than twice a week?

10. Do you season all food salty?

11. Do you have salty soup less than twice a day?

12. Do you have all-noodle soup?

13. Do you have food preserved in salt less than three times a week?

14. Do you use salty sauce before checking the taste?

15. Do you have salty pickles less than twice a day?

16. Do you have vegetables or seaweed at every meal?

17. Do you have fruits every day?

18. Do you have soy products every day?

19. Do you consume dairy products every day?

were free from hypertension, hypercholesterolemia and diabetes at baseline and received a dietary behavior survey again the next year. The range of the concordance rate of each question was from 73.1 (dietary behavior concerned with meat intake) to $89.2 \%$ (dietary behavior concerned with breakfast intake).

\section{Statistical analysis}

The follow-up period was calculated from the day of the first cardiovascular risk survey (baseline survey) to the day of the end-point survey. For subjects who were diagnosed with hypertension, we defined the end-point survey as the survey in which the subject was first diagnosed with hypertension. For subjects who were consistently diagnosed as normotensive, we defined the end-point survey as the last survey.

We prepared 19 dietary behaviors and divided the subjects into four groups according to the answers provided in the baseline and end-point questionnaires. Subjects who answered 'yes' in both the baseline and end-point questionnaires were assigned to group 1. Subjects who answered 'yes' in the baseline questionnaire and 'no' in the end-point questionnaire were assigned to group 2. Subjects who answered 'no' in the baseline questionnaire and 'yes' in the end-point questionnaire were placed in group 3, and subjects who answered 'no' in both the baseline and end-point questionnaires were placed in group 4 on the questions $1,3,4-9,11,13$ and 15-19. As for questions 2,10 , 12 and 14, the subjects were placed in the four groups based on answers opposite to the rules showing above.

Age-adjusted and multivariate-adjusted means and the magnitude of confounding variables were calculated and tested using an analysis of covariance. We calculated the odds ratios (ORs) and 95\% confidence intervals (CIs) by using the logistic regression model for age-adjusted ORs and multivariate-adjusted ORs for the development of hypertension. We used group 1 as the reference group. We used the baseline age, job, body mass index, daily alcohol intake, smoking habits, estimated glomerular filtration rate and systolic blood pressure level at the baseline survey as the confounding variables.

Although there were 19 items in the questionnaire, we listed the results of only 5 items in the tables for better presentation. The results of the other 14 items are listed in Supplementary Table 1.

Furthermore, we examined the differences in the baseline characteristics of 3486 subjects who followed up and 1142 subjects who did not follow-up. The results are reported in Supplementary Table 2.

We used the SAS version 9.1.3 software (SAS Institute Inc., Cary, NC, USA) for all analyses. $P$-values $<0.05$ were considered statistically significant (on two-tailed analyses). 


\section{RESULTS}

During an average 4.6-year follow-up for the 3486 subjects, 846 incident cases $(24.3 \%)$ of hypertension were documented. Table 2 lists the characteristics of the subjects in the first cardiovascular survey according to the five dietary behaviors that were significantly associated with the risk of hypertension. Subjects who consistently did not have one or more egg every day at both the baseline and endpoint surveys (group 4) had a higher diastolic blood pressure

Table 2 Baseline characteristics of subjects according to the answers given to the questionnaires of the baseline and end-point surveys

Answers to questionnaires (baseline/end point)

Yes/Yes (group 1) Yes/No (group 2) No/Yes (group 3) No/No (group 4) Lack of answers

Subjects who had one or more eggs every day

Number and percentage of subjects

Age (years)

Body mass index $\left(\mathrm{kg} \mathrm{m}^{-2}\right)^{\mathrm{a}}$

Alcohol intake (g ethanol per day) ${ }^{a}$

Current smokers (\%)

Systolic blood pressure $(\mathrm{mm} \mathrm{Hg})^{\mathrm{b}}$

Diastolic blood pressure $(\mathrm{mm} \mathrm{Hg})^{\mathrm{b}}$

Estimated glomerular filtration rate $\left(\mathrm{ml} \mathrm{min}^{-1} 1.73 \mathrm{~m}^{-2}\right)^{\mathrm{b}}$

$800(23 \%)$
41.7
23.6
25.6
47
116.7
73.0
82.5

$539(15 \%)$
42.0
23.5
23.7
49
116.9
73.4
83.3

$406(12 \%)$
42.1
23.3
$20.7^{* *}$
42
116.5
72.8
82.0

$1740(50 \%)$
44.0
$23.1^{* *}$
$19.4^{* *}$
47
116.5
$73.8^{*}$
$81.4^{*}$

$1(0 \%)$

Subjects who had meat frequently

Number and percentage of subjects

Age (years)

Body mass index $\left(\mathrm{kg} \mathrm{m}^{-2}\right)^{\mathrm{a}}$

$872(25 \%)$
39.5
23.5
23.1
47
116.8
73.5
82.0

$518(15 \%)$
42.2
23.5
23.5
51
116.7
74.0
81.9

$485(14 \%)$
41.9
23.4
20.8
42
116.7
73.2
81.7

$1606(46 \%)$
45.3
$23.0^{* *}$
$20.6^{*}$
46
116.4
73.4
82.3

Alcohol intake (g ethanol per day) ${ }^{a}$

Current smokers (\%)

Systolic blood pressure $(\mathrm{mm} \mathrm{Hg})^{\mathrm{b}}$

Estimated glomerular filtration rate $\left(\mathrm{ml} \mathrm{min}^{-1} 1.73 \mathrm{~m}^{-2}\right)^{\mathrm{b}}$

$1945(56 \%)$
43.2
23.0
19.7
44
116.6
73.5
81.9

$235(7 \%)$
42.9
23.0
$24.0^{\star}$
45
117.0
73.4
83.1

$444(13 \%)$
42.8
$23.7^{\text {**}}$
$23.5^{\star *}$
47
117.1
73.6
81.6

$861(25 \%)$
42.4
$23.7^{\star \star}$
$24.5^{\star *}$
$52^{* *}$
116.1
73.3
82.3

Alcohol intake (g ethanol per day) ${ }^{a}$

Current smokers (\%)

Systolic blood pressure $(\mathrm{mm} \mathrm{Hg})^{b}$

Diastolic blood pressure $(\mathrm{mm} \mathrm{Hg})^{\mathrm{b}}$

Estimated glomerular filtration rate $\left(\mathrm{ml} \mathrm{min}^{-1} 1.73 \mathrm{~m}^{-2}\right)^{\mathrm{b}}$

$1214(35 \%)$
43.6
23.4
18.5
36
116.4
73.1
81.5

$443(13 \%)$
41.8
23.2
20.0
38
117.0
73.6
81.6

$419(12 \%)$
44.0
23.2
20.9
$48^{* *}$
116.8
73.1
80.9

$1408(40 \%)$
42.4
$23.2^{*}$
$25.1^{* *}$
$58^{* *}$
116.6
$73.8^{*}$
$83.0^{* *}$

Subjects who ate between meals or before bedtime every day

Number and percentage of subjects

Age (years)

$\begin{array}{cc}297(9 \%) & 237(7 \%) \\ 41.8 & 40.6 \\ 23.4 & 23.7 \\ 12.9 & 16.9 \\ 34 & 46^{* *} \\ 115.7 & 116.5 \\ 72.7 & 72.9 \\ 81.4 & 82.9\end{array}$

$285(8 \%)$
42.6
$22.9^{*}$
13.9
$44^{*}$
115.9
72.2
83.4

$\begin{array}{cc}2663(76 \%) & 4(0 \%) \\ 43.3 & 48.5 \\ 23.2 & 25.3 \\ 23.9^{* *} & 17.6 \\ 48^{* *} & 25 \\ 116.8 & 128.8^{*} \\ 73.7^{*} & 80.0 \\ 81.9 & 87.6\end{array}$

${ }^{*} P<0.05$ and ${ }^{* *} P<0.01$

adjusted for age (years).

${ }^{b}$ Adjusted for age (years), body mass index ( $\mathrm{kg} \mathrm{m}^{-2}$ ), ethanol intake (g per day) and current smoking (yes or no). 
compared with subjects who consistently did (group 1, $P=0.03$ ). Subjects who did not consistently consume dairy products every day (group 4) had a higher diastolic blood pressure compared with subjects who consistently did (group $1, P=0.03$ ). Subjects who did not consistently eat between meals or before bedtime every day (group 4) had a higher systolic blood pressure compared with subjects who consistently did (group 1, $P=0.04$ ).

Table 3 shows the age-adjusted and multivariate-adjusted ORs of the risk of hypertension according to the above five dietary behaviors that showed statistical significance. After adjusting for the confounding variables, the OR values were significantly higher in subjects who consistently did not eat meat frequently (group 4) than subjects who consistently did eat meat frequently (group 1) $(\mathrm{OR}=1.32,95 \% \mathrm{CI}: 1.05-1.65 ; P=0.02)$. Regarding dairy products, the OR values were higher in subjects who consistently did not consume dairy products every day (group 4) and who changed their dietary behavior from consuming dairy products every day to not (group 2) than those who consistently did consume dairy products (group 1) $(\mathrm{OR}=1.37,95 \% \mathrm{CI}: 1.11-1.68 ; P=0.003$ and $\mathrm{OR}=1.43$, 95\% CI: $1.08-1.89 ; P=0.01$, respectively). The OR values were higher in subjects who consistently did not eat between meals or before bedtime every day (group 4) than those who consistently did (group 1) $(\mathrm{OR}=1.41,95 \% \mathrm{CI}: 1.00-1.98 ; P=0.05)$. The $\mathrm{OR}$ values were higher in subjects who changed their dietary behaviors from eating one or more eggs every day to not eating eggs (group 2) than those who did consistently eat one or more eggs every day (group 1) $(\mathrm{OR}=1.37,95 \% \mathrm{CI}: 1.04-1.80 ; P=0.02)$. The OR values were higher in subjects who changed their dietary behavior from consuming noodle soup to not consuming noddle soup (group 3) than those who did not consistently consume noodle soup (group 1$)(\mathrm{OR}=1.32$, 95\% CI: $1.03-1.70 ; P=0.03$ ).

Table 4 shows the multivariate-adjusted ORs and 95\% CIs for the risk of hypertension after further adjustment for each dietary behavior listed in Table 2. The associations between dietary behaviors and the risk of hypertension did not change significantly, but the association with eating between meals or before bedtime was no longer statistically significant $(P=0.09)$.

We also calculated the age-adjusted and multivariate-adjusted ORs of the risk of hypertension according to the dietary behaviors that did not show statistical significance. Dietary behaviors characterized by not eating breakfast, eating a meal just before bedtime, not eating fried food, adding salt to meals, using a salty sauce before checking the taste, not having vegetables or seaweed at every meal and not eating fruits led to a higher risk of hypertension, whereas dietary

Table 3 Age-adjusted and multivariate-adjusted ORs of the development of hypertension according to the answers given to the questionnaires in the baseline and end-point surveys

Answers to questionnaires (baseline/end point)

\begin{tabular}{|c|c|c|c|c|}
\hline & \multicolumn{4}{|c|}{ Answers to questionnaires (baseline/end point) } \\
\hline & Yes/Yes (group 1) & Yes/No (group 2) & No/Yes (group 3) & No/No (group 4) \\
\hline \multicolumn{5}{|l|}{ Subjects who had one or more eggs every day } \\
\hline Number of subjects & 6 & 539 & 406 & 1740 \\
\hline Development of hypertension (\%) & 24 & 28 & 22 & 24 \\
\hline Age-adjusted OR and $95 \% \mathrm{Cl}$ & 1.00 & $1.22(0.95-1.57)$ & $0.90(0.68-1.20)$ & $0.91(0.75-1.11)$ \\
\hline Multivariate-adjusted $\mathrm{OR}$ and $95 \% \mathrm{Cl}$ & 1.00 & $1.37(1.04-1.80)$ & $1.08(0.79-1.48)$ & $1.19(0.95-1.48)$ \\
\hline \multicolumn{5}{|l|}{ Subjects who had meat frequently } \\
\hline Number of subjects & 872 & 518 & 485 & 1606 \\
\hline Development of hypertension (\%) & 21 & 23 & 23 & 27 \\
\hline Age-adjusted OR and $95 \% \mathrm{Cl}$ & 1.00 & $1.02(0.79-1.33)$ & $1.01(0.78-1.33)$ & $1.15(0.94-1.41)$ \\
\hline Multivariate-adjusted $\mathrm{OR}$ and $95 \% \mathrm{Cl}$ & 1.00 & $1.02(0.77-1.36)$ & $1.05(0.78-1.41)$ & $1.32(1.05-1.65)$ \\
\hline \multicolumn{5}{|l|}{ Subjects who did not have all-noodle soup } \\
\hline Number of subjects & 1945 & 235 & 444 & 861 \\
\hline Development of hypertension (\%) & 23 & 21 & 32 & 24 \\
\hline Age-adjusted OR and $95 \% \mathrm{Cl}$ & 1.00 & $0.90(0.65-1.26)$ & $1.57(1.25-1.97)$ & $1.05(0.87-1.27)$ \\
\hline Multivariate-adjusted OR and $95 \% \mathrm{Cl}$ & 1.00 & $0.83(0.58-1.19)$ & $1.32(1.03-1.70)$ & $0.94(0.76-1.16)$ \\
\hline \multicolumn{5}{|c|}{ Subjects who consumed dairy products every day } \\
\hline Number of subjects & 1214 & 443 & 419 & 1408 \\
\hline Development of hypertension (\%) & 21 & 26 & 25 & 26 \\
\hline Age-adjusted OR and $95 \% \mathrm{Cl}$ & 1.00 & $1.44(1.12-1.86)$ & $1.24(0.95-1.61)$ & $1.41(1.18-1.70)$ \\
\hline Multivariate-adjusted $\mathrm{OR}$ and $95 \% \mathrm{Cl}$ & 1.00 & $1.42(1.08-1.88)$ & $1.16(0.87-1.55)$ & $1.36(1.11-1.67)$ \\
\hline \multicolumn{5}{|c|}{ Subjects who ate between meals or before bedtime every day } \\
\hline Number of subjects & 297 & 237 & 284 & 2658 \\
\hline Development of hypertension (\%) & 18 & 20 & 21 & 26 \\
\hline Age-adjusted OR and $95 \% \mathrm{Cl}$ & 1.00 & $1.24(0.80-1.92)$ & $1.26(0.83-1.90)$ & $1.57(1.15-2.14)$ \\
\hline Multivariate-adjusted $\mathrm{OR}$ and $95 \% \mathrm{Cl}$ & 1.00 & $1.11(0.69-1.78)$ & $1.38(0.88-2.17)$ & $1.41(1.00-1.98)$ \\
\hline
\end{tabular}

Abbreviations: $\mathrm{Cl}$, confidence interval; OR, odds ratio.

Adjusted for age (years), job, body mass index ( $\mathrm{kgm}^{-2}$ ), ethanol intake ( $\mathrm{g}$ per day), current smoking (yes or no), estimated glomerular filtration rate ( $\mathrm{ml} \mathrm{min}^{-1} 1.73 \mathrm{~m}^{-2}$ ) and systolic blood pressure level from baseline survey $(\mathrm{mm} \mathrm{Hg})$. 
Table 4 Multivariate-adjusted odds ratios of the development of hypertension after further adjustment for dietary patterns

Answers to questionnaires (baseline/end point)

\begin{tabular}{|c|c|c|c|c|}
\hline & Yes/Yes (group 1) & Yes/No (group 2) & No/Yes (group 3) & No/No (group 4) \\
\hline OR and $95 \% \mathrm{Cl}$ & 1.00 & $1.33(1.00-1.75)$ & $1.07(0.78-1.46)$ & $1.11(0.89-1.40)$ \\
\hline \multicolumn{5}{|c|}{ Subjects who had meat frequently } \\
\hline OR and $95 \% \mathrm{Cl}$ & 1.00 & $0.98(0.73-1.31)$ & $0.99(0.74-1.34)$ & $1.26(1.00-1.59)$ \\
\hline \multicolumn{5}{|c|}{ Subjects who consumed dairy products every day } \\
\hline $\mathrm{OR}$ and $95 \% \mathrm{Cl}$ & 1.00 & $1.43(1.07-1.89)$ & $1.16(0.87-1.55)$ & $1.39(1.13-1.71)$ \\
\hline \multicolumn{5}{|c|}{ Subjects who ate between meals or before bedtime every day } \\
\hline OR and $95 \% \mathrm{Cl}$ & 1.00 & $1.07(0.66-1.72)$ & $1.37(0.87-2.16)$ & $1.35(0.96-1.90)$ \\
\hline
\end{tabular}

Abbreviations: $\mathrm{Cl}$, confidence interval; $\mathrm{OR}$, odds ratio.

Adjusted for age (years), job, body mass index $\left(\mathrm{kg} \mathrm{m}^{-2}\right.$ ), ethanol intake ( $\mathrm{g}$ per day), current smoking (yes or no), estimated glomerular filtration rate ( $\mathrm{ml} \mathrm{min}^{-1} 1.73 \mathrm{~m}^{-2}$ ), systolic blood pressure level from baseline survey $(\mathrm{mm} \mathrm{Hg})$ and other dietary behaviors.

behaviors characterized by consuming salty soup, eating foods preserved in salt, eating salty pickles and avoiding soy products led to a lower risk of hypertension. Dietary behaviors characterized by eating until full, consuming soft drinks and having fish or shellfish were not significantly associated with hypertension.

\section{DISCUSSION}

The main finding of our study of Japanese male workers was that the dietary behavior of eating meat and the daily intake of dairy products were inversely associated with the development of hypertension, even after adjusting for other dietary behaviors. Refraining from eating one or more eggs or having noodle soup was positively associated with the development of hypertension.

To our knowledge, no epidemiological study has reported a significant association between meat intake and the risk of hypertension. The Mediterranean diet and the DASH diet recommend a lower intake of red meat to prevent hypertension. ${ }^{5-10}$ However, in the present study, subjects who did not eat meat frequently demonstrated a $29 \%$ higher risk of hypertension compared with subjects who had meat frequently.

As for dairy product intake, several European and US epidemiological studies reported the association between dairy product intake and the risk of hypertension. ${ }^{16-18}$ Among middle-aged and elderly females in the United States, the highest and median quintiles of dairy product intake (2.99-22.1 and 1.40-1.92 servings per day, respectively) showed a $14 \%$ and $7 \%$ lower risk of hypertension, respectively, compared with the lowest quintile ( $0-0.85$ servings per day). ${ }^{16}$ Among young overweight US adults, the lowest category of dairy product intake (0-9 times per week) showed a three-fold higher incidence of hypertension compared with the top category of dairy product intake ( $>35$ times per week). ${ }^{17}$ Among Dutch males and females aged $>55$ years, the highest quartile of dairy product intake (median: $691 \mathrm{~g}$ per day) showed a $24 \%$ lower incidence of hypertension compared with the lowest quartile ( $164 \mathrm{~g}$ per day). ${ }^{18}$ In the present study, subjects who did not consume dairy products every day at the baseline and end-point surveys had a 36\% higher risk of hypertension, and those who stopped consuming dairy products every day between the baseline and end-point surveys had a $44 \%$ higher risk of hypertension compared with the subjects who consumed dairy products every day at baseline and end-point surveys. This result implies that the regular intake of dairy products seems to prevent hypertension. Our study is the first to show the association between dairy products and the development of hypertension in an Asian population.

The mechanism of the inverse association of meat and dairy product intake with hypertension merits some discussion. Specific amino acids that are rich in animal products, such as arginine, taurine, tryptophan and tyrosine, are involved in the control of the vascular system. For example, L-arginine is a vasodilator and substrate of nitric oxide. In a human experiment, an infusion of L-arginine produced an immediate reduction in the blood pressure. ${ }^{19}$ Taurine seems to affect the central nervous system. In an animal experiment, taurine infusion into the brain ventricles lowered blood pressure, ${ }^{20}$ and a human experiment demonstrated that supplemental intake of taurine at $6 \mathrm{~g}$ per day for 7 days lowered blood pressure levels. ${ }^{21}$ Tryptophan and tyrosine also seem to affect the central nervous system by enhancing the synthesis of serotonin, as demonstrated in animal experiments; ${ }^{22,23}$ however, there is no evidence for a similar effect in humans. Although eggs and fish also contain these specific amino acids, they are frequently seasoned with salt in Japan. Therefore, dietary behaviors related to egg and fish intake did not show a significant inverse association with the risk of hypertension. As for dairy products, other mechanisms may exist. Milk peptides have antihypertensive activity by inhibiting angiotensin-1-converting enzyme. ${ }^{24}$ Calcium and magnesium intake is inversely associated with blood pressure levels. ${ }^{8,25}$

With regard to noodle soup, which has a high sodium content, subjects who changed their dietary behavior from having noodle soup to avoiding noodle soup showed a higher risk of hypertension, although subjects who had an all-noodle soup diet did not consistently show a higher risk of hypertension. We suppose the reason was that subjects who developed high blood pressure levels among subjects who had an all-noodle soup diet at the baseline survey were careful to reduce sodium intake and stopped the all-noodle soup diet before the end-point survey. 
Subjects who changed their dietary behavior from consuming one or more eggs every day to avoiding eggs showed a higher risk of hypertension, although subjects who did not consistently consume one or more eggs every day showed a statistically insignificant higher risk of hypertension. We suppose the reason was that subjects who stopped consuming eggs every day lowered their protein intake, which led to an increase in blood pressure. In a recent randomized trial of protein supplementation, in which egg protein formed $20 \%$ of the total protein intake, increased protein intake lowered blood pressure levels. ${ }^{26}$

Based on the results of the present study, Japanese people should consume meat and dairy products frequently to prevent hypertension. The recommendation of high meat intake is different from the DASH diet. However, a previous cross-sectional study of Japanese subjects showed an inverse association between animal protein intake and blood pressure levels, ${ }^{27}$ which adds support to the notion that Japanese people should consume meat.

The strength of the present study is that the methodology was superior to that of previous cross-sectional studies of Japanese populations. ${ }^{12,28}$ Prospective studies have little informational bias, and their results are more revealing than those of cross-sectional studies. The results of prospective studies also reinforce the causal relationships between risk factors and the development of hypertension more clearly than those of crosssectional studies.

The limitations of the present study warrant discussion. First, we were unable to obtain the precise date of the development of hypertension because our analysis was based on information from annual cardiovascular risk checks. We then used logistic regression analysis to calculate the ORs based on information from the baseline questionnaire. Second, our questionnaire only allowed subjects to choose answers 'yes' or 'no,' which made it difficult to evaluate the dose-response association between each type of dietary behavior and the development of hypertension. Furthermore, we did not determine the validity of the questionnaire fully. Only questions concerning sodium intake were validated. ${ }^{29,30}$ However, we previously compared our questionnaire with other validated food frequency questionnaires and found that the subjects who reported eating something frequently had higher intake than the other subjects, ${ }^{31}$ which may add some support to the validity of the questionnaire. Third, we used the first blood pressure measurement in the present study due to the low number of subjects in whom blood pressure levels were measured twice. However, inclusion of the second measurement in the analysis, when available, did not change the results. In addition, we used systolic blood pressure levels measured at baseline in the multiple logistic regression analysis. We confirmed that the results did not change when we used diastolic instead of systolic blood pressure levels. Fourth, because of poor follow-up, we excluded from the analyses 1142 subjects who had a potentially higher risk of hypertension because of age, blood pressure levels and estimated glomerular filtration rate. Fifth, the present study included only males, and the results cannot be applied to females.

In conclusion, our prospective study of Japanese male workers showed that the intake of meat, dairy products and eggs was inversely associated with the risk of hypertension, whereas the intake of noodle soup was positively associated with the risk of hypertension. These results point to a beneficial dietary behavior that can prevent hypertension in male Japanese workers. Further epidemiological studies and clinical trials are necessary to establish the best dietary behavior for the prevention of hypertension among Japanese males.

\section{CONFLICT OF INTEREST}

The authors declare no conflict of interest.

\section{ACKNOWLEDGEMENTS}

We acknowledge the contributions of public health nurses, dietitians, nurses, medical technologists and other staff of the Osaka Medical Center for Health Science and Promotion.

1 Lawes CM, Bennett DA, Parag V, Woodward M, Whitlock G, Lam TH, Suh I, Rodgers A Asia Pacific Cohort Studies Collaboration. Blood pressure indices and cardiovascular disease in the Asia Pacific region: a pooled analysis. Hypertension 2003; 42: 69-75.

2 Imano H, Kitamura A, Sato S, Kiyama M, Ohira T, Yamagishi K, Noda H, Tanigawa T, Iso $\mathrm{H}$, Shimamoto T. Trends for blood pressure and its contribution to stroke incidence in the middle-aged Japanese population: the Circulatory Risk in Communities Study (CIRCS). Stroke 2009; 40: 1571-1577.

3 Ikeda A, Iso H, Yamagishi K, Inoue M, Tsugane S. Blood pressure and the risk of stroke, cardiovascular disease, and all-cause mortality among Japanese: the JPHC Study. Am J Hypertens 2009; 22: 273-280.

4 Lewington S, Clarke R, Qizilbash N, Peto R, Collins R. Prospective Studies Collaboration. Age-specific relevance of usual blood pressure to vascular mortality: a metaanalysis of individual data for one million adults in 61 prospective studies. Lancet 2002; 360: 1903-1913.

5 Appel LJ, Champagne CM, Harsha DW, Cooper LS, Obarzanek E, Elmer PJ, Stevens VJ, Vollmer WM, Lin PH, Svetkey LP, Stedman SW, Young DR, Writing Group of the PREMIER Collaborative Research Group. Effects of comprehensive lifestyle modification on blood pressure control: main results of the PREMIER clinical trial. JAMA 2003; 289: 2083-2093.

6 Forman JP, Stampfer MJ, Curhan GC. Diet and lifestyle risk factors associated with incident hypertension in women. JAMA 2009; 302: 401-411.

7 Núñez-Córdoba JM, Valencia-Serrano F, Toledo E, Alonso A, Martínez-González MA. The Mediterranean diet and incidence of hypertension: the Seguimiento Universidad de Navarra (SUN) Study. Am J Epidemiol 2009; 169: 339-346.

8 Ascherio A, Hennekens C, Willett WC, Sacks F, Rosner B, Manson J, Witteman J, Stampfer MJ. Prospective study of nutritional factors, blood pressure, and hypertension among US women. Hypertension 1996; 27: 1065-1072.

9 Schulze MB, Hoffmann K, Kroke A, Boeing H. Risk of hypertension among women in the EPIC-Potsdam Study: comparison of relative risk estimates for exploratory and hypothesis-oriented dietary patterns. Am J Epidemiol 2003; 158: 365-373.

10 Appel LJ, Moore TJ, Obarzanek E, Vollmer WM, Svetkey LP, Sacks FM, Bray GA Vogt TM, Cutler JA, Windhauser MM, Lin PH, Karanja N. A clinical trial of the effects of dietary patterns on blood pressure DASH Collaborative Research Group. N Engl J Med 1997; 336: 1117-1124.

11 Lee SA, Cai H, Yang G, Xu WH, Zheng W, Li H, Gao YT, Xiang YB, Shu XO. Dietary patterns and blood pressure among middle-aged and elderly Chinese men in Shanghai. Br J Nutr 2010; 104: 265-275.

12 Utsugi MT, Ohkubo T, Kikuya M, Kurimoto A, Sato RI, Suzuki K, Metoki H, Hara A Tsubono Y, Imai Y. Fruit and vegetable consumption and the risk of hypertension determined by self measurement of blood pressure at home: the Ohasama study. Hypertens Res 2008; 31: 1435-1443.

13 Ogihara T, Kikuchi K, Matsuoka H, Fujita T, Higaki J, Horiuchi M, Imai Y, Imaizumi T, Ito S, Iwao H, Kario K, Kawano Y, Kim-Mitsuyama S, Kimura G, Matsubara H, Matsuura H, Naruse M, Saito I, Shimada K, Shimamoto K, Suzuki H, Takishita S, Tanahashi N, Tsuchihashi T, Uchiyama M, Ueda S, Ueshima H, Umemura S, Ishimitsu T, Rakugi H, Japanese Society of Hypertension Committee. The Japanese Society of Hypertension Guidelines for the Management of Hypertension (JSH 2009). Hypertens Res 2009; 32: 3-107.

14 Matsuo S, Imai E, Horio M, Yasuda Y, Tomita K, Nitta K, Yamagata K, Tomino Y, Yokoyama $\mathrm{H}$, Hishida A, Collaborators developing the Japanese equation for estimated GFR. Revised equations for estimated GFR from serum creatinine in Japan. Am J Kidney Dis 2009; 53: 982-992.

$15 \mathrm{~A}$ committee on health assessment. Health assessment manual. Kousei Kagaku Kenkyusyo, Tokyo, Japan, 2000, in Japanese.

16 Wang L, Manson JE, Buring JE, Lee IM, Sesso HD. Dietary intake of dairy products, calcium, and vitamin $\mathrm{D}$ and the risk of hypertension in middle-aged and older women. Hypertension 2008; 51: 1073-1079.

17 Pereira MA, Jacobs Jr DR, Van Horn L, Slattery ML, Kartashov AI, Ludwig DS. Dairy consumption, obesity, and the insulin resistance syndrome in young adults: the CARDIA Study. JAMA 2002; 287: 2081-2089.

18 Engberick MF, Hendriksen MAH, Schoutem EG, van Rooij FJ, Hofman A, Witteman JC, Geleijnse JM. Inverse association between dairy intake and hypertension: the Rotterdam Study. Am J Clin Nutr 2009; 89: 1877-1883.

19 Hishikawa K, Nakaki T, Suzuki H, Kato R, Saruta T. Role of L-arginine-nitric oxide pathway in hypertension. J Hypertens 1993; 11: 639-645.

20 Sgaragli G, Pavan F. Effects of amino acid compounds injected into cerebrospinal fluid spaces, on colonic temperature, arterial blood pressure and behaviour of the rat. Neuropharmacology 1972; 11: 45-56. 
21 Fujita T, Ando K, Noda H, Ito Y, Sato Y. Effects of increased adrenomedullary activity and taurine in young patients with borderline hypertension. Circulation 1987; 75 525-532.

22 Sved AF, van Itallie CM, Fernstrom JD. Studies on the antihypertensive action of L-tryptophan. J Pharmacol Exp Ther 1982; 221: 329-333.

23 Sved AF, Fernstrom JD, Wurtman RJ. Tyrosine administration reduces blood pressure and enhances brain norepinephrine release in spontaneously hypertensive rats. Proc Natl Acad Sci USA 1979; 76: 3511-3514.

24 Jauhiainen T, Korpela R. Milk peptides and blood pressure. J Nutr 2007; 137 825S-829S.

25 Iso H, Terao A, Kitamura A, Sato S, Naito Y, Kiyama M, Tanigaki M, lida M, Konishi M, Shimamoto T. Calcium intake and blood pressure in seven Japanese populations. Am J Epidemiol 1991; 133: 776-783.

26 Teunissen-Beekman KF, Dopheide J, Geleijnse JM, Bakker SJ, Brink EJ, de Leeuw PW, van Baak MA. Protein supplementation lowers blood pressure in overweight adults: effect of dietary proteins on blood pressure (PROPRES), a randomized trial. Am J Clin Nutr 2012: 95: 966-971.

27 Umesawa M, Sato S, Imano H, Kitamura A, Shimamoto T, Yamagishi K, Tanigawa T, Iso H. Relations between protein intake and blood pressure in Japanese men and women: the Circulatory Risk in Communities Study (CIRCS). Am J Clin Nutr 2009; 90 377-384.

28 Miura K, Okuda N, Turin TC, Takashima N, Nakagawa H, Nakamura K, Yoshita K, Okayama A, Ueshima H, NIPPON DATA80/90 Research Group. Dietary salt intake and blood pressure in a representative Japanese population: baseline analyses of NIPPON DATA80. J Epidemiol 2010; 20: S524-S530.

29 Iso H, Harada S, Shimamoto T, Sato S, Kitamura A, Sankai T, Tanigawa T, lida M, Komachi Y. Angiotensinogen T174M and M235T variants, sodium intake and hypertension among non-drinking, lean Japanese men and women. J Hypertens 2000; 18: 1197-1206.

30 Iso H, Shimamoto T, Yokota K, Sankai T, Jacobs Jr DR, Komachi Y. Community-based education classes for hypertension control: a 1.5-year randomized controlled trial. Hypertension 1996; 27: 968-974.

31 Nishimura S, Miyazaki J, Kuwabara C, Hatano M, Hakui T, Asai M, Nagano A, Kurokawa M, Kitamura A, Sato S, Shimamoto T. Conditions and problems of dietary behavior in urban populations (Part 2)-Relationship between dietary research and dietary behaviors. Nihon Koshu Eisei Zasshi 2006; 57(Suppl): 956 (in Japanese).

Supplementary Information accompanies the paper on Hypertension Research website (http://www.nature.com/hr) 